\title{
Nevus Sebaceus of Jadassohn - Rare Cutaneous Hamartoma in Infancy
}

\author{
${ }^{1}$ Venkata Ramana Poondla, ${ }^{2}$ Srilakshmi Kothakoona, ${ }^{1}$ Venkata Janardana Rao \\ Kakulapati, \\ ${ }^{I}$ (Department of Pediatric surgery, Andhra Medical College, Visakhapatnam, Andhra Pradesh, India) \\ ${ }^{2}$ (Department of Anaesthesiology, Andhra Medical College, Visakhapatnam, Andhra Pradesh, India)
}

\begin{abstract}
Congenital hamartomas in infancy embrace cutaneous, Vascular and Lymphatic malformations. Nevus sebaceus of Jadassohn is a rare cutaneous lesion over face in infancy but it is a common epidermal hamartoma of scalp in pre pubertal and adult age groups. The lesions have a potential for benign and malignant transformation under hormonal influence particularly at puberty. Surgical excision is indicated primarily for cosmetic reasons and secondly if there is any suspicion of neoplastic changes.
\end{abstract}

Keywords: Hamartoma, Nevus sebaceus, Secondary tumors

\section{Introduction}

The term Nevus sebaceus was first introduced by Jadassohn in 1895 to describe a congenital Hamartoma on the scalp or face showing an excess of sebaceous glands(1). They are usually of sporadic occurrence and are seen in an estimated $0.3 \%$ of neonates. The natural history of the lesion is described in three stages like Pre pubertal, Post pubertal and neoplastic change. Secondary tumors are reported (2) usually in middle age with a risk of $5 \%$. Treatment Modalities include $\mathrm{CO}_{2}$ laser therapy, Photodynamic therapy, Excision and reconstruction.

\section{Case Report}

An 8 month old female child was brought in view of a rapidly growing swelling over right side of face since birth. Parents noticed the lesion measuring $0.5 \mathrm{~cm}$ in diameter at birth which attained a size of $10 \mathrm{x} 8 \mathrm{~cm}$ at the time of consultation ("Fig." 1 ).The lesion has a broad pedicle, exophytic and with a honey comb appearance of the surface ("Fig." 2 ), and confined to the skin and subcutaneous tissue with no regional lymph node involvement. Facial Nerve is not infiltrated clinically. The child underwent Wide excision of the lesion under general anesthesia with endotracheal intubation. Good approximation of the wound was achieved ("Fig."3).The excised tumor was sent for Histopathological Examination.

\section{Histopathology}

The cut section of the specimen was grey white to pale yellow in color with nodular surface having areas of necrosis. Microscopic appearance composed of hamartomatous, conglomerate large sebaceous glands associated with defective hair follicles, Acanthosis and Papillomatosis ("Fig."4). Dermis shows multiple follicular cysts with central keratin material surrounded by lobules of pilo-sebaceous glands. Cellular features are unremarkable. Deeper part of the stroma is fibrotic in nature. The Histopathological features are consistent with Nevus sebaceus with a differential diagnosis of Steatocystoma Multiplex.Sutures removed on the fifth postoperative day and wound healed well. One year post-operative follow up revealed no recurrence of the tumor.

\section{Conclusion}

Epidermal naevi are subdivided into keratinocytic and organoid naevi, the latter includes Naevus Sebaceus (NS). Histology shows disordered sebaceous glands, hair follicles, and apocrine glands. Differential diagnosis includes cutis aplasia, verrucae vulgaris, and linear epidermal nevus. During puberty, most lesions become prominent with neoplastic changes in sebaceous and apocrine components. Secondary tumours may occur in around 25\% of NS (3) ("TABLE" 1). Development of an exophytic nodule on a Nevus Sebaceus usually represents a benign appendageal tumor or viral wart. Rapid, Circumscribed enlargement or ulceration should arouse suspicion of malignant transformation. A variety of appendageal tumors, sometimes multiple, may develop within sebaceous naevi. The most commonly reported secondary tumors are Syringocystadenoma papilliferum and Trichoblastoma (4). Elective excision remains the most common treatment approach because of cosmetic concerns and the potential for growth, particularly under hormonal stimulation in puberty (5).Treatment options include surgical excision in preadolescence under local anesthesia versus removal in infancy under general anesthesia. The latter is preferable for large, cosmetically disfiguring lesions which may 
require reconstructive procedures. Excision was planned in the index case mainly due to cosmetic reason and to prevent development of secondary tumors at puberty. Recently, somatic mosaicism has been shown, with activating RAS mutations in HRAS or KRAS in NS lesional keratinocytes. The recent identification of key epidermal signaling abnormalities underlying the cell proliferation means that future development of new medical treatments for NS that target the aberrant signaling pathways may also be feasible(6).

\section{Figures And Tables}

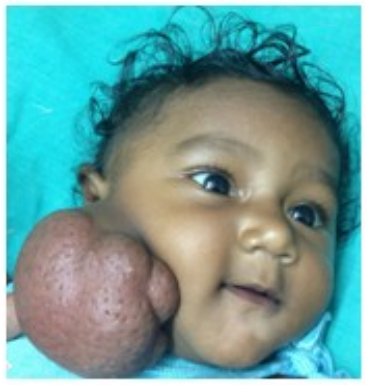

Fig.l Nevus Sebaceus of an Infant

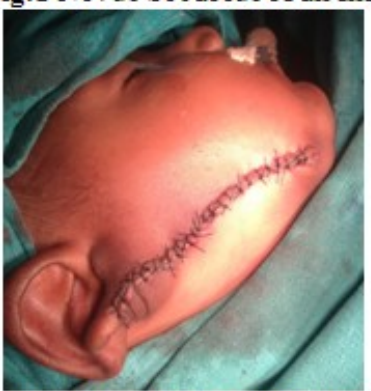

Fig.3 Intra Operative Photo

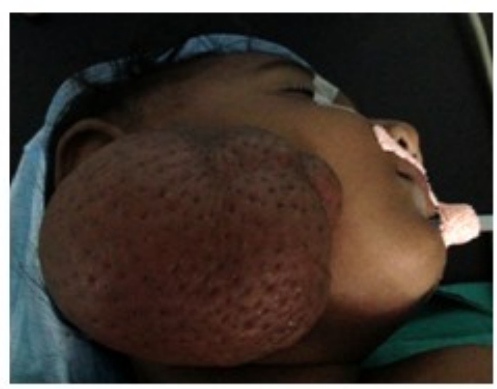

Fig.2 Honey comb appearance of NS

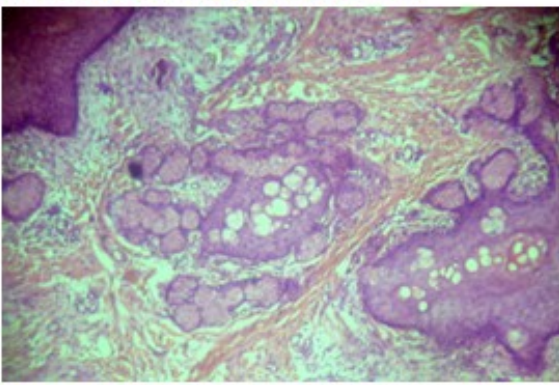

Fig.4 Histology showing Conglomerate Sebaceous Glands and Hyperkeratosis

Table1: Secondary tumors in Nevus Sebaceus

\begin{tabular}{|l|l|}
\hline \multicolumn{1}{|c|}{ Benign Tumors } & \multicolumn{1}{c|}{ Malignant Tumors } \\
\hline 1.Syringocystadenoma Papilliferum & Basal Cell Carcinoma \\
\hline 2. Syringoma & Syringocystadenoma Papilliferum \\
\hline 3. Apocrine Cystadenoma & Trichoblastoma \\
\hline 4. Trichilemmoma & Viral Warts \\
\hline 5. Spiradenoma & Keratoacanthoma \\
\hline 6. Nodular Hidradenoma & Malignant Melanoma \\
\hline 7. Keratoacanthoma & Squamous Carcinoma \\
\hline
\end{tabular}

Acknowledgements

Authors acknowledge the immense help received from the scholars whose articles are cited and included in references of this manuscript. The authors are also grateful to authors / editors / publishers of all those articles, journals and books from where the literature for this article has been reviewed and discussed.

\section{References}

[1]. Mehregan AH, Pinkus H. Life history of organoid nevi.Special reference to nevus sebaceous of Jadassohn. ArchDermatol 1965; 91:574-588

[2]. Etienne Wang, Joyce Siong. Carcinomatous changes like Sebaceous carcinoma, Trichoblastoma, and Poroma arising from a nevus Journal of Cutaneous Pathology, Volume 40, Issue 7, July 2013, Pages: 676- 682.

[3]. Cribier B, Scrivener Y, Grosshans E. Tumors arising in nevus sebaceus: a study of 596 cases. J Am Acad Dermatol 2000; 42:263268.

[4]. Munir H. Idriss, Dirk M. Elston, Secondary neoplasms associated with nevus sebaceus of Jadassohn: A study of 707 cases, Journal of the American Academy of Dermatology, 2014, 70, 2, 332

[5]. Margulis A, Bauer BS, Corcoran JF.Surgical management in cutaneous manifestations of linear nevus sebaceus syndrome. Plast Reconstr Surg 2003; 111:1043-1050.

[6]. Happle R, lethal genes surviving by mosaicism: a possible explanation for sporadic birth defects involving the skin, American Academy of Dermatology 1987; 16: 899-906. 